EPJ Web of Conferences 59, 07004 (2013)

DOI: $10.1051 /$ epjconf/20135907004

(C) Owned by the authors, published by EDP Sciences, 2013

\title{
Development of high-energy fiber CPA system
}

\author{
J. Ogino ${ }^{1,4, a}$, K. Sueda ${ }^{2,4}$, T. Kurita ${ }^{3,4}$, T. Kawashima ${ }^{3,4}$ \\ and N. Miyanaga ${ }^{1,4}$ \\ ${ }^{1}$ Institute of Laser Engineering, Osaka University, 2-6 Yamada-oka, Suita, Osaka, Japan \\ ${ }^{2}$ Photon Pioneers Center, Osaka University, 2-1 Yamada-oka, Suita, Osaka, Japan \\ ${ }^{3}$ Hamamatsu Photonics K. K., 5000 Hirakuchi, Hamakita, Shizuoka, Japan \\ ${ }^{4}$ JST CREST, Sanbancho 5, Chiyoda-ku, Tokyo, Japan
}

\begin{abstract}
An intense broadband laser system based on a Nd:glass chirped pulse amplifier (CPA) requires a stable front end with good beam quality. To prepare such a front end without using a regenerative amplifier or optical parametric chirped pulse amplification (OPCPA) pumped by the second harmonic of a Nd:YAG laser, we have developed a fiber CPA system using laser diode (LD)-pumped single-mode fibers, largemode-area (LMA) fibers, and photonic-crystal (rod) fibers. The output pulse energy achieved was $1.2 \mathrm{~mJ}$ at a central wavelength of $1053 \mathrm{~nm}$ with a spectral width of $10 \mathrm{~nm}$. The power fluctuation was reduced to $0.4 \%$ rms at a repetition rate of $10 \mathrm{kHz}$. The output of this fiber laser is useful as a pump source for a few-cycle noncollinear optical parametric amplifier (NOPA) and a seed for a LD-pumped high-power Nd:glass CPA system.
\end{abstract}

\section{INTRODUCTION}

There has been growing interest in intense femtosecond pulses with durations of less than $10 \mathrm{fs}$ for a variety of applications such as attosecond science using high-order harmonics [1-3]. An ultrashort, intense laser system with a peak power TW class can be realized by using a noncollinear optical parametric amplifier (NOPA) [4-8]. As such, we are developing a near-infrared (NIR) few-cycle NOPA system, of which the designed specifications are a 5-fs pulse width and a 30-TW peak power at a $10-\mathrm{Hz}$ repetition rate. The important issues in designing such an ultrashort pulse laser system are the ultrabroadband NOPA technique, the dispersion control, and the carrier-envelope phase (CEP) stabilization. To realize state-of-art technology in these areas, the quality and stability of the beam are essential. High power femtosecond lasers generally use the method of chirped pulse amplification (CPA) [9]. For this purpose, instead of a using a regenerative amplifier or an OPA pumped by the second harmonic of a commercially available Nd:YAG laser, we have developed a high-energy fiber CPA system with a central wavelength of $1053 \mathrm{~nm}$. This system can be used both as a sub-picosecond pump source for the preamplifier of a NOPA and as a seed pulse for a laser diode (LD)-pumped Nd:glass CPA laser [10], which can be used as a pump source for the main amplifier of an intense few-cycle NOPA laser.

\section{DESIGN OF HIGH-ENERGY FIBER CPA SYSTEM}

The fiber CPA system is designed to deliver an output pulse energy of 1-2 mJ with a spectral width of $\sim 10 \mathrm{~nm}$, which ensures that there is enough gain in the preamplifier of the NOPA and that there

\footnotetext{
ae-mail: ogino-j@ile.osaka-u.ac.jp
}

This is an Open Access article distributed under the terms of the Creative Commons Attribution License 2.0, which permits unrestricted use, distribution, and reproduction in any medium, provided the original work is properly cited. 


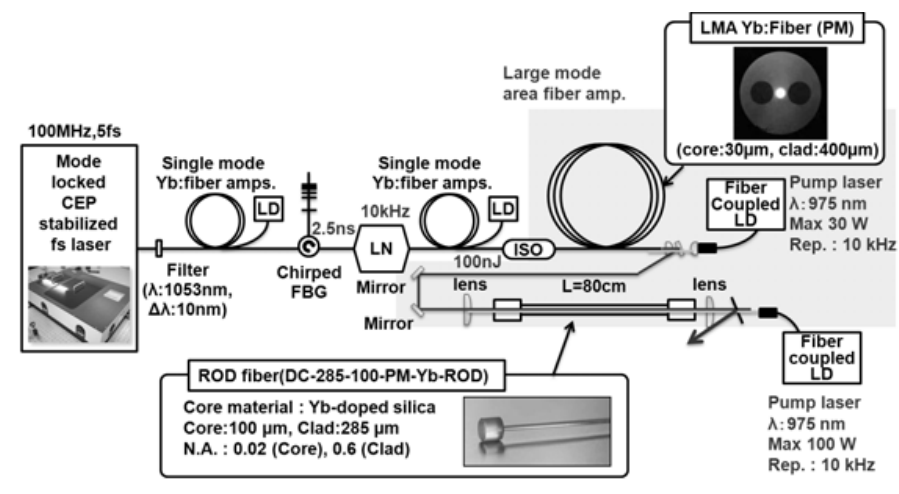

Figure 1. System layout of the all-fiber CPA system.

is efficient energy extraction in the LD-pumped Nd:glass laser. Figure 1 shows the optical layout of the laser system, which consists of a broadband few-cycle Ti:sapphire oscillator, a chirped fiber Bragg grating (CFBG) stretcher, a Li niobate (LN) modulator as a pulse picker, Yb-doped single-mode fiber and large-mode-area (LMA) fiber as preamplifiers, a Yb-doped photonic-crystal (rod) fiber as a main amplifier, and a bulk diffraction grating compressor. The output of the oscillator was split into two parts. One of them was used as a seed signal of the NOPA (not shown in Figure 1), and the other was injected into the fiber CPA system. The oscillator delivered a 200-mW pulse train with a spectrum ranging from $650 \mathrm{~nm}$ to $1100 \mathrm{~nm}$ at a 100-MHz repetition rate. The infrared edge of the spectrum overlapped with the gain band of the $\mathrm{Yb}$-doped fiber lasers, including the spectral gain region of the Nd:glass amplifier. At the injection point to the fiber system, a band-pass filter was set to extract 1053-nm light with a 10-nm spectral width (full width at half maximum [FWHM]). The pulses were stretched in a CFBG to $2.5 \mathrm{~ns}$ with the 10-nm bandwidth. An LN modulator was used to reduce the pulse repetition rate to $10 \mathrm{kHz}$. The pulses were preamplified in polarization-maintaining Yb-doped single-mode fiber and LMA fiber, which also suppressed the amplified spontaneous emission (ASE) noise. The length and core diameter of the LMA fiber were $4 \mathrm{~m}$ and $30 \mu \mathrm{m}$, respectively. The pump source of the LMA fiber was a fibercoupled quasi-continuous-wave (QCW) LD with a maximum power of $30 \mathrm{~W}$ at a 975 -nm wavelength. The main amplifier was constructed using a polarization-maintaining, air-clad, photonic-crystal, rodtype fiber with a core diameter of $100 \mu \mathrm{m}$ (DC-285-100-PM-Yb-ROD, NKT Photonics) [4, 11] pumped by a fiber-coupled QCW LD with a maximum power of $100 \mathrm{~W}$. To reduce the thermal effects in the LMA and rod fibers, the LD pumping was operated at a repetition rate of $10 \mathrm{kHz}$ with a $50 \%$ duty cycle.

\section{LASER CHARACTERISTICS OF THE FIBER CPA SYSTEM}

The pulse seeded to the LMA Yb-doped fiber had an energy of about $100 \mathrm{~nJ}$ and a pulse duration of $2.5 \mathrm{~ns}$. The pump LD was operated in QCW mode $(10-\mathrm{kHz}$ repetition rate with a $50 \%$ duty cycle). Figures 2(a) and (b) show the gain and output pulse energy of the LMA fiber amplifier, respectively, as functions of the average pumping power. When the average pump power was $9.4 \mathrm{~W}$, the maximum gain obtained was 320 , which corresponds to a maximum output energy of $32 \mu \mathrm{J}$. Figures 3(a) and (b) show the gain and output energy of the rod fiber amplifier as functions of the average pumping power. To avoid damage to the fiber, the current operation level of the rod fiber amplifier was limited to a maximum pulse energy of $\sim 1 \mathrm{~mJ}$. Thus, the maximum gain measured was 88 (output pulse energy of $1.2 \mathrm{~mJ}$ with a pulse width of $2.5 \mathrm{~ns}$ FWHM) with an average pump power of $53 \mathrm{~W}$. These measured output pulse energies were in agreement with theoretical estimations, with a discrepancy of $85 \%$. To evaluate the beam quality, a beam pattern was observed on the output surface of the rod fiber. The pattern was nearly Gaussian and had a beam size that was consistent with the mode field diameter of this fiber $(76.2 \mu \mathrm{m})$. 


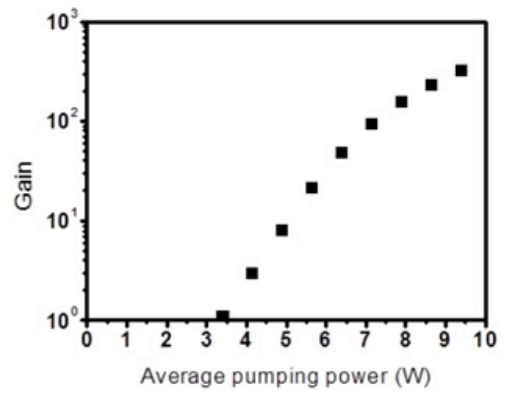

(a)

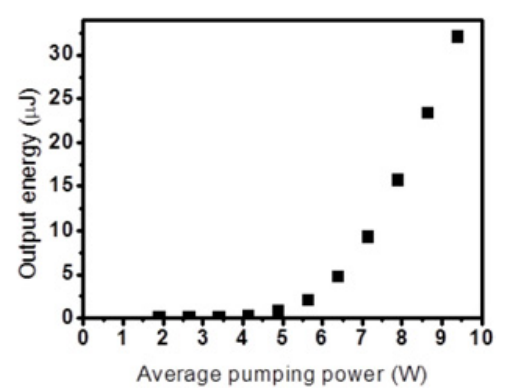

(b)

Figure 2. Gain (a) and output pulse energy (b) characteristics of the LMA fiber amplifier as functions of the average pumping power.

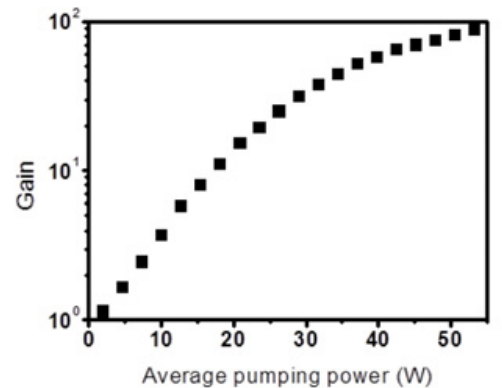

(a)

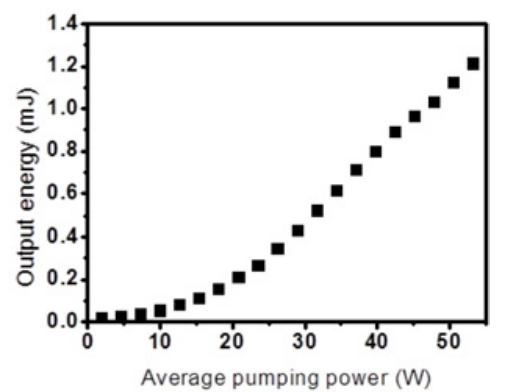

(b)

Figure 3. Gain (a) and output pulse energy (b) characteristics of the rod fiber amplifier as functions of the average pumping power.

We calculated the B-integral value to evaluate the influence of the nonlinear effect. It was estimated to be 0.7 at the output of the rod fiber amplifier. It is found that the nonlinear phase shift has hardly an influence. We measured the temporal features of the output power of the LMA and rod fiber amplifiers. The output power fluctuation of the LMA fiber was $0.3 \% \mathrm{rms}(2.2 \% \mathrm{P}-\mathrm{V})$, and that of rod fiber was $0.4 \% \mathrm{rms}(2.6 \% \mathrm{P}-\mathrm{V})$, which was much better than the power fluctuation $(19 \% \mathrm{rms}$ for equivalent pulse energy) observed for a three-stage optical parametric CPA pumped by a commercial Nd:YAG laser.

\section{PULSE COMPRESSION AND FREQUENCY DOUBLING}

The pulse compressor consists of a pair of diffraction gratings with groove densities of 1480 lines $/ \mathrm{mm}$. The sizes of Grating are $80 \mathrm{~mm} \times 110 \mathrm{~mm}$ (Grating1) and $150 \mathrm{~mm} \times 260 \mathrm{~mm}$ (Grating2). The separation distance between the gratings was set to $4447 \mathrm{~mm}$ with an incident angle of $47^{\circ}$ for a chirping of $2.5 \mathrm{~ns} / 10 \mathrm{~nm}$, and the beam path was arranged in the double-pass configuration using a roof mirror. The pulse duration was measured by an autocorrelator (Pulse Check PM NIR 700-1100 ver. 15, APE), as shown in Figure 4. From a measured autocorrelation trace ( $700 \mathrm{fs}$, FWHM), the pulse width was estimated to be $430 \mathrm{fs}$, assuming a Gaussian shape. The output pulse energy of the compressor was $560 \mu \mathrm{J}$. This pulse was then fed into a 1-mm-thick type-I BBO crystal for the frequency doubling. The pulse energy converted to the $527-\mathrm{nm}$ wavelength was $160 \mu \mathrm{J}$, which corresponds to a conversion efficiency of $29 \%$. 


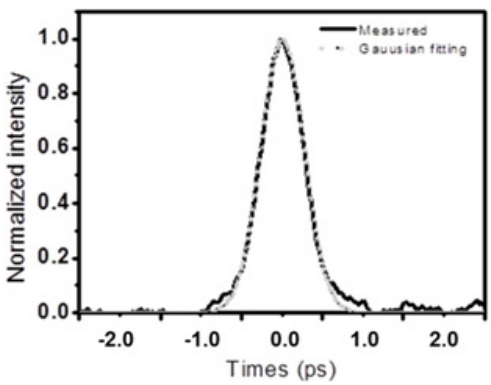

Figure 4. Autocorrelation trace of compressed pulse.

\section{CONCLUSION}

We have constructed a high-energy all-fiber CPA system using single-mode, LMA, and photonic crystal (rod) fibers. This system can deliver an output energy of $1.2 \mathrm{~mJ}$ with a $10-\mathrm{nm}$ spectral width at a $1053-\mathrm{nm}$ central wavelength. The total gain of this system was about $1 \times 105$. The output power fluctuation was $0.4 \% \mathrm{rms}(2.6 \% \mathrm{P}-\mathrm{V})$. The pulses had an energy of $560 \mu \mathrm{J}$ and a duration of $430 \mathrm{fs}$ at a repetition rate of $10 \mathrm{kHz}$ after the pulse compression. The second harmonic pulse energy obtained was $160 \mu \mathrm{J}$ (conversion efficiency of $29 \%$ ). This fiber CPA system will be used as a front end of a higherpower LD-pumped Nd:glass laser, which can be used as a J-class pump source for the main amplifier stage of a NOPA and the pump source of the preamplifier stage of the NOPA.

\section{References}

[1] F. X. Kartner, ed., Few-Cycle Laser Pulse Generation and Its Applications (Springer Verlag, Berlin, 2004)

[2] M. Drescher, et al., Science 291, 1923 (2001)

[3] F. Tavella, et al., Opt. Lett. 32, 2227 (2007)

[4] A. Dubietis, et al., Opt. Commun. 88, 437 (1992)

[5] S. Adachi, et al., Optics Express 16, 14341 (2008)

[6] S. Witte, et al., Opt. Express 13, 4903 (2005)

[7] F. Tavella, et al., Opt. Lett. 32, 2227 (2007)

[8] D. Herrmann, et al, Opt. Lett. 34, 2459 (2009)

[9] D. Strickland, et al, Opt. Commun. 56, 219 (1985)

[10] F. Röser, et al., Opt. Lett. 32, 3495 (2007)

[11] J. Boullet, et al., Opt. Express 16, 17891 (2008) 